\title{
Minimum Variance Beamforming for High Frame-Rate Ultrasound Imaging
}

\author{
Holfort, Iben Kraglund; Gran, Fredrik; Jensen, Jørgen Arendt
}

Published in:

Proceedings of IEEE Ultrasonics Symposium

Link to article, DOI:

10.1109/ULTSYM.2007.388

Publication date:

2007

Document Version

Publisher's PDF, also known as Version of record

Link back to DTU Orbit

Citation (APA):

Holfort, I. K., Gran, F., \& Jensen, J. A. (2007). Minimum Variance Beamforming for High Frame-Rate Ultrasound Imaging. In Proceedings of IEEE Ultrasonics Symposium (pp. 1541-1544). IEEE.

https://doi.org/10.1109/ULTSYM.2007.388

\section{General rights}

Copyright and moral rights for the publications made accessible in the public portal are retained by the authors and/or other copyright owners and it is a condition of accessing publications that users recognise and abide by the legal requirements associated with these rights.

- Users may download and print one copy of any publication from the public portal for the purpose of private study or research.

- You may not further distribute the material or use it for any profit-making activity or commercial gain

- You may freely distribute the URL identifying the publication in the public portal 


\title{
Minimum Variance Beamforming for High Frame-Rate Ultrasound Imaging
}

\author{
Iben Kraglund Holfort, Fredrik Gran and Jørgen Arendt Jensen \\ Center for Fast Ultrasound Imaging, Ørsted•DTU, Bldg. 349, \\ Technical University of Denmark, DK-2800 Kgs. Lyngby, Denmark
}

\begin{abstract}
This paper investigates the application of adaptive beamforming in medical ultrasound imaging. A minimum variance (MV) approach for near-field beamforming of broadband data is proposed. The approach is implemented in the frequency domain, and it provides a set of adapted, complex apodization weights for each frequency sub-band. As opposed to the conventional, Delay and Sum (DS) beamformer, this approach is dependent on the specific data.

The performance of the proposed MV beamformer is tested on simulated synthetic aperture (SA) ultrasound data, obtained using Field II. For the simulations, a $7 \mathrm{MHz}$, 128-element, phased array transducer with $\lambda / 2$-spacing was used. Data is obtained using a single element as the transmitting aperture and all $128 \mathrm{el}$ ements as the receiving aperture. A full SA sequence consisting of 128 emissions was simulated by sliding the active transmitting element across the array. Data for 13 point targets and a circular cyst with a radius of $5 \mathrm{~mm}$ were simulated. The performance of the MV beamformer is compared to DS using boxcar weights and Hanning weights, and is quantified by the Full Width at Half Maximum (FWHM) and the peak-side-lobe level (PSL). Single emission \{DS Boxcar, DS Hanning, MV \} provide a PSL of $\{-16,-36,-49\} \mathrm{dB}$ and a FWHM of $\{0.79,1.33,0.08\} \mathrm{mm}$ $=\{3.59 \lambda, 6.05 \lambda, 0.36 \lambda\}$. Using all 128 emissions, $\{$ DS Boxcar, DS Hanning, MV $\}$ provide a PSL of $\{-32,-49,-65\} \mathrm{dB}$, and a FWHM of $\{0.63,0.97,0.08\} \mathrm{mm}=\{2.86 \lambda, 4.41 \lambda, 0.36 \lambda\}$. The contrast of the beamformed single emission responses of the circular cyst were calculated to $\{-18,-37,-40\} \mathrm{dB}$.

The simulations have shown that the frequency sub-band MV beamformer provides a significant increase in lateral resolution compared to DS, even when using considerably fewer emissions. An increase in resolution is seen when using only one single emission. Furthermore, it is seen that an increase of the number of emissions does not alter the FWHM. Thus, the MV beamformer introduces the possibility for high frame-rate imaging with increased resolution.
\end{abstract}

\section{INTRODUCTION}

Recently, the application of adaptive beamforming methods to the field of medical ultrasound imaging has been an increasingly area of interest. In recent literature [1]-[6] adaptive beamformers have been applied to medical ultrasound imaging with significant improvements in terms of lateral resolution and contrast.

In traditional beamforming, the Delay and Sum (DS) beamformer uses a fixed, predefined set of apodization weights. Whereas the adaptive methods actively finds a set of apodization weights, which is adapted to the specific data.

One of these adaptive methods is the Minimum Variance (MV) beamformer, which finds a set of weights that minimizes the variance of the weighted sensor signals under the constraint that the signal emerging from the point of interest is passed without distortion. The MV optimized weights are found in a single iteration, but it does require a matrix inversion, which increases the computational cost compared to DS.

In this paper an approach for near-field beamforming of broad-band data is proposed. This approach is implemented in the frequency domain, and it provides a set of adapted, complex apodization weights for each frequency sub-band.

\section{METHOD}

\section{A. Presteering}

As in conventional beamforming, the sensor signals are presteered, so that each scan line is dynamically focused. Considering a linear array transducer with $M$ sensor elements, the $m$ th dynamically focused sensor signal along the $\ell$ th scan line is given by

$$
y_{m, \ell}(z)=s\left(\frac{\left\|\vec{r}_{\ell}^{(x m t)}(z)\right\|+\left\|\vec{r}_{m, \ell}^{(r c v)}(z)\right\|}{c}\right)
$$

for $m=0,1, \ldots, M-1$ and $\ell=0,1, \ldots, L-1$, where $z$ denotes the spatial position along the $\ell$ th scan line, $s(t)$ is the received waveform, $\vec{r}^{(x m t)}$ and $\vec{r}(r c v)$ are the spatial positions of the transmitting and the receiving sensor elements, and $c$ is the speed of sound.

The output of the beamformer is given by the weighted sum of the dynamically focused scan lines, so that the $\ell$ th scan line is given by

$$
b_{\ell}(z)=\sum_{m=0}^{M-1} w_{m, \ell} y_{m, \ell}(z),
$$

where $w_{m, \ell}$ is the apodization weight for the $m$ th sensor signal.

\section{B. Sub-Band Beamforming}

The MV beamformer [7] is originally developed for narrowband applications. Applying MV to broad-band ultrasound data, the sensor signals are divided into sub-bands using the short-time Fourier transform. For each point, $z_{0}$, along the $\ell$ th scan line, the Fourier transform is applied on a segment of the sensor signals. The $m$ th segmented sensor signal is given by

$$
\tilde{y}_{m, \ell}\left(z, z_{0}\right)=y_{m, \ell}\left(z-z_{0}\right), \quad z \in[-Z / 2 ; Z / 2] \text {, }
$$


where $Z$ is the size of the segment. For the given point, $z_{0}$, the beamformer output for each spatial frequency sub-band, $k$, is given by

$$
B_{\ell}\left(k, z_{0}\right)=\sum_{m=0}^{M-1} w_{m, \ell}^{*}\left(k, z_{0}\right) Y_{m, \ell}\left(k, z_{0}\right),
$$

where $Y_{m, \ell}\left(k, z_{0}\right)$ is the Fourier transform of the $m$ th segmented sensor signal, $\tilde{y}_{m, \ell}\left(z, z_{0}\right)$, given in (3), and $\{\cdot\}^{*}$ denotes the complex conjugate. By defining the vectors

$$
\begin{aligned}
& \mathbf{w}_{\ell}\left(k, z_{0}\right)=\left(\begin{array}{llll}
w_{0, \ell}\left(k, z_{0}\right) & w_{1, \ell}\left(k, z_{0}\right) & \cdots & w_{M-1, \ell}\left(k, z_{0}\right)
\end{array}\right)^{T} \\
& \mathbf{Y}_{\ell}\left(k, z_{0}\right)=\left(\begin{array}{llll}
Y_{0, \ell}\left(k, z_{0}\right) & Y_{1, \ell}\left(k, z_{0}\right) & \cdots & Y_{M-1, \ell}\left(k, z_{0}\right)
\end{array}\right)^{T}
\end{aligned}
$$

the beamformer output (4) rewrites into

$$
B_{\ell}\left(k, z_{0}\right)=\mathbf{w}_{\ell}\left(k, z_{0}\right)^{H} \mathbf{Y}_{\ell}\left(k, z_{0}\right),
$$

where the superscripts, $\{\cdot\}^{T}$ and $\{\cdot\}^{H}$, denote the nonconjugate and the conjugate transpose, respectively.

Note that the sub-band division provides the possibility of weighting both each sub-band and each point differently.

\section{Minimum Variance Beamforming}

The adaptive beamformer uses a set of apodization weights, which are dependent on the frequency content of the specific sensor signals. The MV beamformer continuously updates the weights, so that the variance (or power) of the beamformer output is minimized, while the response from the focus point is passed without distortion. The power of the beamformer output is given by

$$
\begin{aligned}
\mathcal{P}_{\ell}\left(k, z_{0}\right) & =\mathcal{E}\left\{\left|B_{\ell}\left(k, z_{0}\right)\right|^{2}\right\} \\
& =\mathbf{w}_{\ell}\left(k, z_{0}\right)^{H} \mathbf{R}_{\ell}\left(k, z_{0}\right) \mathbf{w}_{\ell}\left(k, z_{0}\right),
\end{aligned}
$$

where $\mathcal{E}\{\cdot\}$ denotes the expectation value, and $\mathbf{R}_{\ell}\left(k, z_{0}\right)$ is the covariance matrix given by

$$
\mathbf{R}_{\ell}\left(k, z_{0}\right)=\mathcal{E}\left\{\mathbf{Y}_{\ell}\left(k, z_{0}\right) \mathbf{Y}_{\ell}\left(k, z_{0}\right)^{H}\right\} \text {. }
$$

Mathematically, the MV beamformer is expressed as [7]

$$
\begin{gathered}
\min _{\mathbf{w}_{\ell}\left(k, z_{0}\right)} \mathbf{w}_{\ell}\left(k, z_{0}\right)^{H} \mathbf{R}_{\ell}\left(k, z_{0}\right) \mathbf{w}_{\ell}\left(k, z_{0}\right) \\
\quad \text { subject to } \mathbf{w}_{\ell}\left(k, z_{0}\right)^{H} \mathbf{e}\left(k, z_{0}\right)=1,
\end{gathered}
$$

where $\mathbf{e}\left(k, z_{0}\right)$ is the so-called steering vector, which characterizes the response from the focus point.

The solution to the optimization problem (9) can be found in a single iteration using Lagrangian multiplier theory as [7]

$$
\mathbf{w}_{\ell}\left(k, z_{0}\right)=\frac{\mathbf{R}_{\ell}\left(k, z_{0}\right)^{-1} \mathbf{e}\left(k, z_{0}\right)}{\mathbf{e}\left(k, z_{0}\right)^{H} \mathbf{R}_{\ell}\left(k, z_{0}\right)^{-1} \mathbf{e}\left(k, z_{0}\right)},
$$

provided that $\mathbf{R}_{\ell}\left(k, z_{0}\right)^{-1}$ exists. Due to presteering and subband division, the response from the focus point will resemble a plane wave incident directly onto the array. Thus, the steering vector is constant across the array and independent on the frequency, and it simply becomes a $M \times 1$-vector of ones.

\section{Subarray Averaging}

In real applications, the covariance matrix is unknown and must be estimated from data. To obtain a useful estimate, the array is divided into overlapping subarrays, and the subcovariance matrices are averaged across the array. According to [8] the spatially smoothed covariance matrix estimate will always become non-singular, if the size of the subarray satisfies $M_{p} \leq \frac{M}{2}$. The covariance matrix estimate can be expressed as

$$
\mathbf{R}_{\ell}\left(k, z_{0}\right)=\sum_{p=0}^{M-M_{p}+1} \mathbf{G}_{p, \ell}\left(k, z_{0}\right) \mathbf{G}_{p, \ell}\left(k, z_{0}\right)^{H},
$$

where $\mathbf{G}_{p, \ell}\left(k, z_{0}\right)$ denotes the $p$ th subarray given by

$$
\mathbf{G}_{p, \ell}\left(k, z_{0}\right)=\left(Y_{p, \ell}\left(k, z_{0}\right) Y_{p+1, \ell}\left(k, z_{0}\right) \cdots Y_{p+M_{p}-1, \ell}\left(k, z_{0}\right)\right)^{T}
$$

for $p=0,1, \ldots, M_{p}-1$. Note that this reduces the dimension of the covariance matrix, and thus the number of weights will be reduced correspondingly.

\section{RESUlTS}

The proposed MV beamformer is tested on simulated synthetic aperture (SA) ultrasound data, obtained using Field II [9], [10]. For the simulations, a $7 \mathrm{MHz}, 128$-element, phased array transducer with $\lambda / 2$-spacing was used. Data is obtained using a single element as the transmitting aperture and all $M=$ 128 elements as the receiving aperture. A full SA sequence consisting of 128 emissions was simulated by sliding the active transmitting element across the array. Data for 13 point targets and a circular cyst with a radius of $5 \mathrm{~mm}$ were simulated.

The MV beamformer is implemented in the frequency domain using the short time Fourier transform with a segment size corresponding to the length of the excitation pulse convolved with the two-way impulse response of the transducer. A subarray size of $M_{p}=\frac{M}{4}=32$ was used, and before beamforming, additional white, Gaussian noise with a signalto-noise ratio (SNR) of $60 \mathrm{~dB}$ was added to each of the sensor signals.

The performance of MV is compared to DS using boxcar weights and Hanning weights. The performance is quantified by the Full Width at Half Maximum (FWHM) and the peakside-lobe level (PSL), which is defined as the peak value of the first side-lobe.

\section{A. Point Targets}

The beamformed responses of the 13 point targets are shown in Fig. 1(a)-(d) with a dynamic range of $-50 \mathrm{~dB}$. The three responses in Fig. 1(a)-(c) are averaged over 128 emissions, and the MV response on the right, Fig. 1(d), is from a single element emission (corresponding to element \#64). The lateral variation of the beamformed responses at $z=\{40,45\} \mathrm{mm}$ are seen in Fig. 3(a)-(b). It is seen that MV provides a significant increase of the lateral resolution and a lowering of the sidelobes.

The resolution and contrast improvements are quantified using the FWHM and PSL. These quantitative measures are calculated at a depth of $40 \mathrm{~mm}$ and are given in Table I. The 


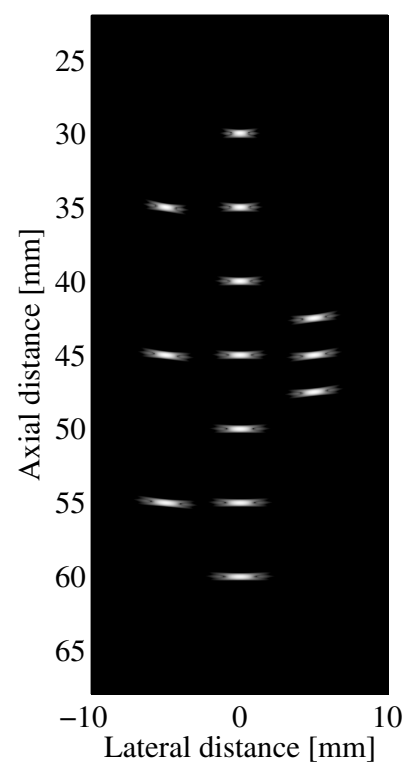

(a) DS, Boxcar

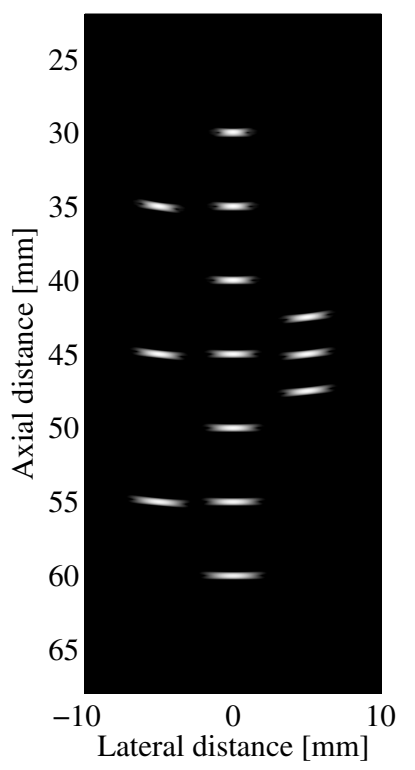

(b) DS, Hanning

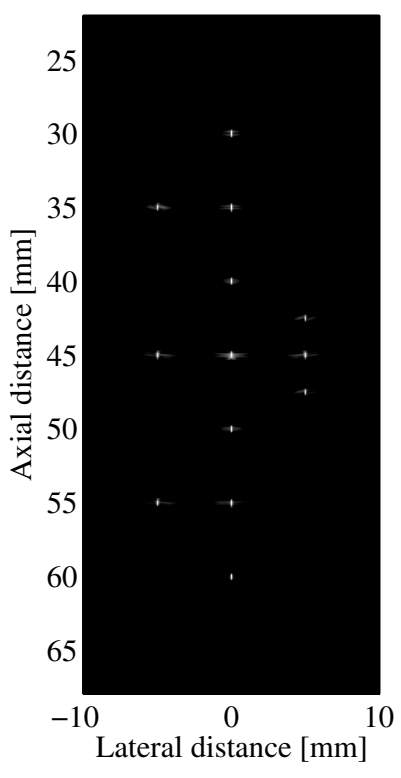

(c) MV

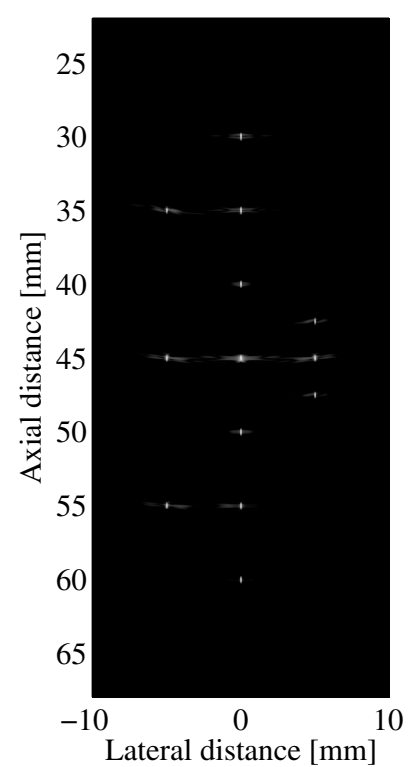

(d) MV, single element

Fig. 1. Beamformed responses of the 13 point targets. (a)-(c) The images are averaged over 128 emissions. (d) No averaging is applied, response from a single element emission (element \#64). All images are shown with a dynamic range of $-50 \mathrm{~dB}$.

measures are given for the single element emission and for the full SA sequence. It is seen that the MV beamformer provides a significant improvement in terms of both FWHM and PSL. The FWHM of MV from a single emission response comprise only $\{12.7 \%, 8.2 \%\}$ of the FWHM from the full DS sequence using DS $\{$ Boxcar,Hanning $\}$.

The MV response does not change significantly in the interval of $[-40 ; 0] \mathrm{dB}$. The FWHM is calculated at a level of $-6 \mathrm{~dB}$ and will not change for $\mathrm{MV}$, when averaging over all 128 emissions. This is shown in Fig. 2(a), where the FWHM for DS and MV are shown with respect to the number of emissions. Furthermore, the ratio of energy above

(a)

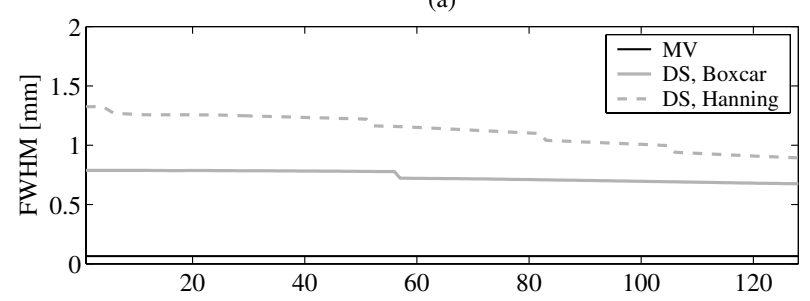

(b)

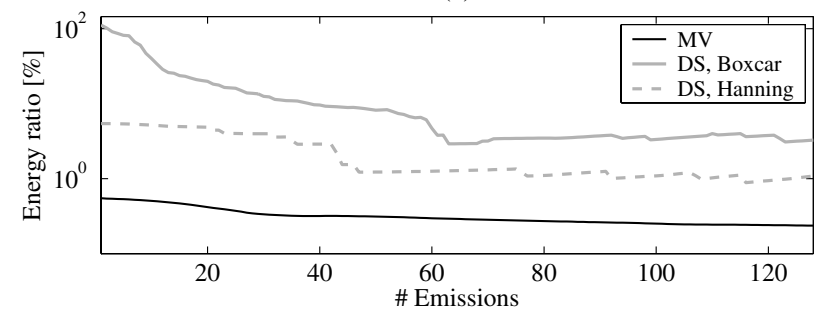

Fig. 2. Full Width at Half Maximum (FWHM) and the energy ratio above and below $-40 \mathrm{~dB}$ of the beamformed responses with respect to the number of emissions.

\begin{tabular}{|c|c|c|c|}
\hline & PSL & \multicolumn{2}{|c|}{ FWHM } \\
\hline \multicolumn{4}{|c|}{ Single emission (element \#64) } \\
\hline DS, Boxcar & $-16 \mathrm{~dB}$ & $0.79 \mathrm{~mm}$ & $3.59 \lambda$ \\
\hline DS, Hanning & $-36 \mathrm{~dB}$ & $1.33 \mathrm{~mm}$ & $6.05 \lambda$ \\
\hline MV & $-49 \mathrm{~dB}$ & $0.08 \mathrm{~mm}$ & $0.36 \lambda$ \\
\hline \multicolumn{4}{|c|}{ Full sequence (averaged over 128 emissions) } \\
\hline DS, Boxcar & $-32 \mathrm{~dB}$ & $0.63 \mathrm{~mm}$ & $2.86 \lambda$ \\
\hline DS, Hanning & $-49 \mathrm{~dB}$ & $0.97 \mathrm{~mm}$ & $4.41 \lambda$ \\
\hline MV & $-65 \mathrm{~dB}$ & $0.08 \mathrm{~mm}$ & $0.36 \lambda$ \\
\hline
\end{tabular}

FUll Width at Half MAXimum (FWHM) AND PEAK-SIDE-LOBE LEVEL (PSL) FOR THE BEAMFORMED RESPONSES AT $z=40 \mathrm{MM}$.
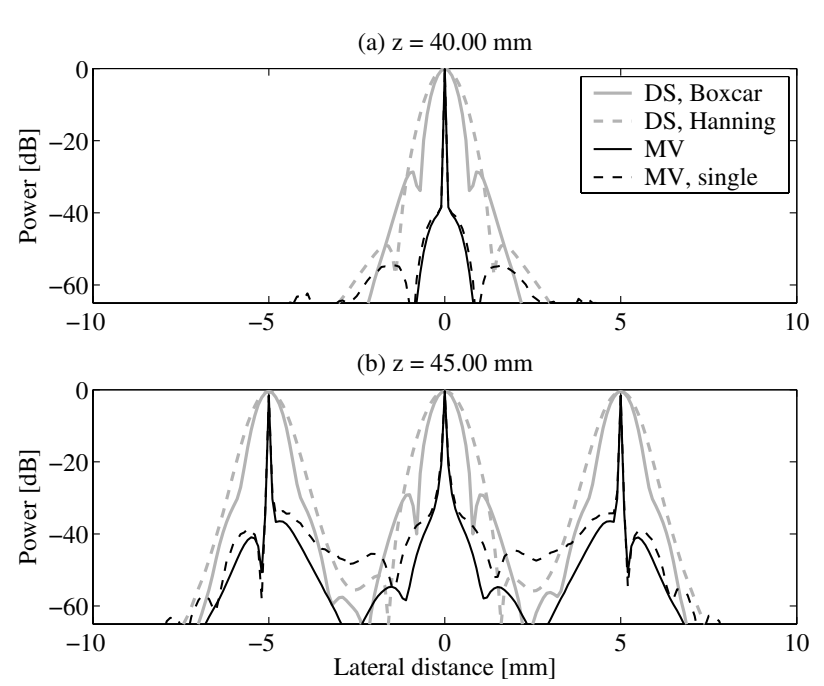

Fig. 3. Lateral variation at $z=\{40,45\} \mathrm{mm}$ of the beamformed responses shown in Fig. 1(a)-(d). 


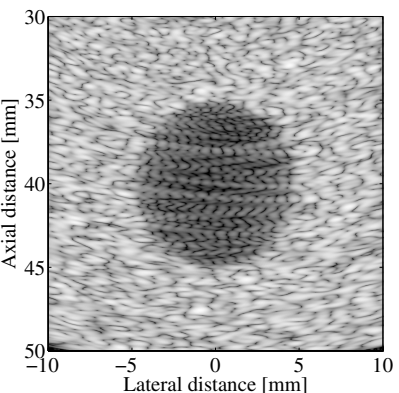

(a) DS, Boxcar Contrast: $-18 \mathrm{~dB}$

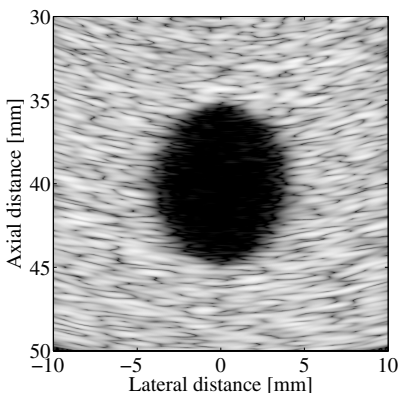

(b) DS, Hanning Contrast: $-37 \mathrm{~dB}$

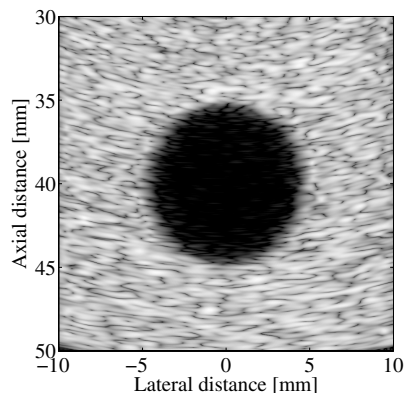

(c) MV Contrast: $-40 \mathrm{~dB}$

Fig. 4. Circular cyst with radius of $5 \mathrm{~mm}$ and center at $(x, z)=(0,40) \mathrm{mm}$. (a)-(c) The images are responses to a single element emission (element \#64). All images are shown with a dynamic range of $-50 \mathrm{~dB}$.

and below $-40 \mathrm{~dB}$ has been calculated relative to the number of emissions, shown in Fig. 2(b). This ratio is a measure of the relative energy within the main-lobe. As it reduces, the relative energy within the main-lobe reduces, which could mean that the width of the main-lobe decreases. It is seen that this ratio reduces with the number of emissions. However, the reduction for MV is not as immense as for DS. Thus, the MV response does not improve significantly, when averaging over several emissions.

\section{B. Circular Cyst}

In this section, simulated data of a circular cyst in a speckle pattern is considered. The circular cyst has a radius of $5 \mathrm{~mm}$ and center at $(x, z)=(0,40) \mathrm{mm}$. The speckle pattern is simulated with 10 randomly placed scatterers within a resolution cell of $\lambda^{3}$ to ensure fully developed speckle.

The beamformed responses from a single element emission (corresponding to element \#64) are shown in Fig. 4(a)-(c). The resulting contrast for each of the three responses are $\{-18,-37,-40\} \mathrm{dB}$. The contrast is computed as the ratio between the mean energy within the cyst and outside the cyst.

In real applications, the DS responses will be averaged over several emissions to obtain a better contrast. Furthermore, averaging emissions from different spatial positions would also result in a circular cyst using DS with Hanning weights. However, these single emission responses are shown to emphasize the differences between the three beamforming approaches.

It is seen that the high side-lobe level of the DS Boxcar apodization results in a rather poor contrast. Furthermore, it is seen that the DS response using Hanning apodization does not result in a circular shape of the cyst. The shape is rather oval due to the lateral broadening from the Hanning apodization. However, the Hanning apodization provides a suitable contrast level, as the side-lobes are lowered to the SNR level at $-60 \mathrm{~dB}$. The MV response shows a circular shape of the cyst as well as a suitable contrast difference between the inner part of the cyst and the speckle pattern. From this investigation, it is seen that the MV beamformer provides a lowering of the side-lobe level without smoothing the responses laterally.

\section{CONCLUSiOnS}

The proposed approach for near-field, broad band adaptive beamforming was implemented in the frequency domain and investigated based on simulated ultrasound data. The investigations have shown that each frequency sub-band can be treated independently. The adaptive sub-band beamformer provides a significant increase in lateral resolution and contrast compared to the conventional beamformer, even when using considerably fewer emissions. An increase in resolution is seen when using only one single emission. Furthermore, it is seen that an increase of the number of emissions does not alter the FWHM. Thus, the MV beamformer introduces the possibility for high frame-rate imaging with increased resolution.

\section{ACKNOWLEDGMENT}

This work was supported by grant 9700883, 9700563 and 2601-0178 from the Danish Science Foundation and by B-K Medical A/S, Denmark.

\section{REFERENCES}

[1] M. Sasso and C. Cohen-Bacrie, "Medical Ultrasound Imaging Using the Fully Adaptive Beamformer," in Proc. IEEE Int. Conf. Acous., Speech, Sig. Pro., March 2005, vol. 2, pp. 489-492.

[2] J. A. Mann and W. F. Walker, "A Constrained Adaptive Beamformer for Medical Ultrasound: Initial Results," in Proc. IEEE Ultrason. Symp., Oct. 2002, vol. 2, pp. 1807-1810.

[3] F. Viola and W. F. Walker, "Adaptive Signal Processing in Medical Ultrasound Beamforming," in Proc. IEEE Ultrason. Symp., 2005, vol. 4, pp. 1980-1983.

[4] J.-F. Synnevåg, A. Austeng, and S. Holm, "Minimum Variance Adaptive Beamforming Applied to Medical Ultrasound Imaging," in Proc. IEEE Ultrason. Symp., Sept. 2005, vol. 2, pp. 1199-1202.

[5] J.-F. Synnevåg, A. Austeng, and S. Holm, "High frame-rate and high resolution medical imaging using adaptive beamforming," in Proc. IEEE Ultrason. Symp., Oct. 2006, vol. 2.

[6] J.-F. Synnevåg, A. Austeng, and S. Holm, "Adaptive Beamforming Applied to Medical Ultrasound Imaging," IEEE Trans. Ultrason., Ferroelec., Freq. Contr., vol. 54, no. 8, pp. 1606-1613, August 2007.

[7] J. Capon, "High-Resolution Frequency-Wavenumber Spectrum Analysis," Proc. IEEE, vol. 57, no. 8, pp. 1408-1418, August 1969.

[8] T.-J. Shan and T. Kailath, "Adaptive Beamforming for Coherent Signals and Interference," IEEE Trans. Acous., Speech, Sig. Pro., vol. 33, no. 3 , pp. 527-536, June 1985 .

[9] J. A. Jensen and N. B. Svendsen, "Calculation of pressure fields from arbitrarily shaped, apodized, and excited ultrasound transducers," IEEE Trans. Ultrason., Ferroelec., Freq. Contr., vol. 39, pp. 262-267, 1992.

[10] J. A. Jensen, "Field: A program for simulating ultrasound systems," Med. Biol. Eng. Comp., vol. 10th Nordic-Baltic Conference on Biomedical Imaging, Vol. 4, Supplement 1, Part 1, pp. 351-353, 1996b. 